Conclusion These simple recommendations, supported by a real-life research example, can improve sensitivity analyses for unmeasured confounding and reduce the potential for selective reporting, thereby improving the quality of population health research.

\section{P92 CAUSAL INFERENCE-INFORMED RE-ANALYSIS TO GAIN INSIGHTS INTO FACTORS ASSOCIATED WITH DROP-OUT FROM WEIGHT-LOSS PROGRAMMES}

\begin{abstract}
1,2,3 Ridda Ali*, ${ }^{5}$ Andrew J Prestwich, ${ }^{2}$ Jiaqi Ge, ${ }^{1,3,4}$ Mark S Gilthorpe. 'Leeds Institute for Data Analytics, University of Leeds, Leeds, UK; ${ }^{2}$ Faculty of Environment, University of Leeds, Leeds, UK; ${ }^{3}$ Faculty of Medicine and Health, University of Leeds, Leeds, UK; ${ }^{4}$ The Alan Turing Institute, London, UK; ${ }^{5}$ School of Psychology, University of Leeds, Leeds, UK
\end{abstract}

\subsection{6/jech-2021-SSMabstracts. 178}

Background Understanding the factors that predict/cause individuals' withdrawal, or dropout, from weight loss can provide useful insight into adaptations that could ensure that such programmes have greater impact. If one event follows another, conclusions are drawn that the first event caused the second. However, these associations may be observed due to chance, confounding, or selection bias. Although a lot of research has been conducted to identify factors related to attrition and adherence in weight management/loss programmes, their findings do not have a concrete (causal) interpretation beyond recognising that some predictors are often favoured over others from an initial pool of candidate predictors.

Methods Dalle Grave et al. (2015) recruited 634 patients seeking obesity treatment at Italian medical centres. They performed logistic regression to assess the association between demographic, personality characteristics, eating disorder features, psychological well-being, and attrition. This study aims to illustrate the key issues through directed acyclic graph (DAG) informed re-analysis of the Dalle Grave et al. (2015)'s data to explore if and by how much conclusions might vary between common prediction approaches and a causal inference approach.

Results According to Dalle Grave et al. (2015), personality traits, which were assessed through the Temperament and Character Inventory (TCI), are less relevant in predicting attrition. In contrast, causal inference analysis suggests that temperament scores (harm avoidance (Probability $=0.33$; CI $=0.29$, 0.37 ), novelty seeking (Probability $=0.34$; $\mathrm{CI}=0.30,0.38$ ), persistence (Probability $=0.30 ; \quad \mathrm{CI}=0.26,0.34)$, and reward dependence (Probability $=0.30 ; \mathrm{CI}=0.26,0.33)$ ) and character scores (self-transcendence (Probability $=0.34 ; \mathrm{CI}=0.30,0.39$ ), cooperativeness (Probability $=0.32 ; \mathrm{CI}=0.27,0.36$ ), self-directedness (Probability $=0.32 ; \mathrm{CI}=0.27,0.37)$ ) are causally associated with higher probability of drop-out. Additionally, Dalle Grave et al. (2015) considered body uneasiness scores to be irrelevant in predicting drop-out. Whereas, causal inference analysis indicated that higher body uneasiness scores are causally associated with the highest probability of drop-out (Probability $=0.39$; CI $=0.34,0.44$ ).

Conclusion New insights into factors that predict/cause dropout from weight-loss programmes can be gained through causal inference-informed analysis. On the basis of this reanalysis, factors previously identified as irrelevant or excluded with respect to a traditional prediction perspective appear to be important from a causal perspective. Dalle Grave et al. (2015)'s analysis can be considered a case of the 'table 2' fallacy, where mutually adjusted coefficients in a prediction model are (inappropriately) inferred to have an equivalent interpretation. Different causal models must be generated, based on a DAG, to derive 'correct' (causal) inferences.

\section{P93 THE DANGERS OF CAUSALLY UNAWARE ETHICAL FRAMEWORKS FOR HEALTH DATA}

${ }^{1,2}$ Gabriela Arriagada Bruneau*, ${ }^{1,3,4}$ Georgia Tomova, ${ }^{3,4}$ Peter WG Tennant, ${ }^{3,4}$ Mark S Gilthorpe. 'Leeds Institute for Data Analytics, University of Leeds, Leeds, UK; ${ }^{1}$ Philosophy Department, Inter-disciplinary Applied Ethics Centre, University of Leeds, Leeds, UK; ${ }^{3}$ The Alan Turing Institute, London, UK; ${ }^{4}$ Faculty of Medicine and Health, University of Leeds, Leeds, UK

\subsection{6/jech-2021-SSMabstracts. 179}

Background During the COVID-19 pandemic we have seen various disastrous approaches regarding the use an implementation of measures and studies that performed on past and current health data. Accordingly, in this study, we criticize the lack of conceptual engineering to integrate ethical principles and values into the design and application of data-driven endeavours, with a particular examination at health data. We argue how we cannot strive for a robust ethical assessment without a critically causal framework

Methods Firstly, we analyse the translational gap and conceptual conflation of the terms: 'bias and fairness' and 'transparency and explainability', highlighting the misleading definitions and uses given to these concepts at a technical and ethical level. The main distinctions presented clarify the moral expectations given to these concepts and criticise the insufficient development of a conceptual analysis that targets them. We suggest that a fundamental part of a solution to reduce this translational gap implies embracing and applying a causal framework. Thus, we show why using causal models and, most importantly, a causal narrative cannot only help to prevent unethical effects, but it can also influence the efficiency of prediction models and their outcomes. Efficiency, in this case, transforms into an ethically laden concept that demands a causal narrative to align with ethical principles. Finally, we go through examples of COVID-19 decision-making that could have benefitted from a causal approach, highlighting the negative consequences of the NHS electronic health records platform and an OpenSAFELY publication in Nature that substantially suffers from the Table 2 Fallacy.

Discussion This analysis puts into discussion an interdisciplinary approach to increase critical ethical awareness about fairness. Providing robust and reliable frameworks to analyse and present data, especially in sensitive times like a world pandemic, requires trustworthy practices.

Conclusion Integrating ethics into data-driven solutions cannot be limited by the bias-aware fairness formalisations or the naïve applications of transparency and explainability. When it comes to the real-world application of models, their effects can harm individuals in society. Non-causal approaches tend to dissipate elements of agency and responsibility, which are fundamental to the development of what we can call 'good science'. 